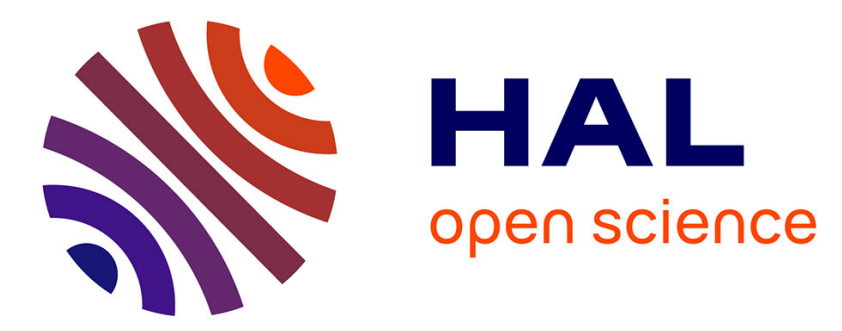

\title{
High-speed footage shows transient ultrasonic nucleation of different hydrophobic particles in suspension
}

\author{
Michiel Postema, Ryunosuke Matsumoto, Ri-Ichiro Shimizu, Albert Thijs \\ Poortinga, Nobuki Kudo
}

\section{- To cite this version:}

Michiel Postema, Ryunosuke Matsumoto, Ri-Ichiro Shimizu, Albert Thijs Poortinga, Nobuki Kudo. High-speed footage shows transient ultrasonic nucleation of different hydrophobic particles in suspension. Japanese Journal of Applied Physics, 2020, 59 (SK), pp.SKKD07. 10.35848/1347-4065/ab7f19 . hal-03192686

\section{HAL Id: hal-03192686 \\ https://hal.science/hal-03192686}

Submitted on 11 Apr 2021

HAL is a multi-disciplinary open access archive for the deposit and dissemination of scientific research documents, whether they are published or not. The documents may come from teaching and research institutions in France or abroad, or from public or private research centers.
L'archive ouverte pluridisciplinaire HAL, est destinée au dépôt et à la diffusion de documents scientifiques de niveau recherche, publiés ou non, émanant des établissements d'enseignement et de recherche français ou étrangers, des laboratoires publics ou privés. 


\title{
High-speed footage shows transient ultrasonic nucleation of different hydrophobic particles in suspension
}

\author{
Michiel Postema ${ }^{1 *}$, Ryonosuke Matsumoto ${ }^{2}$, Ri-ichiro Shimizu ${ }^{2}$, Albert T. Poortinga $^{3}$, and \\ Nobuki Kudo ${ }^{2}$ \\ ${ }^{1}$ School of Electrical and Information Engineering, University of the Witwatersrand, Johannesburg, \\ 1 Jan Smuts Laan, 2001 Braamfontein, South Africa \\ ${ }^{2}$ Faculty of Information Science and Technology, Hokkaido University, Kita 9 Chome, Nishi 14 Jo, Kita-ku, \\ Sapporo, Hokkaido 060-0814, Japan \\ ${ }^{3}$ Department of Mechanical Engineering, Eindhoven University of Technology, De Zaale, 5600 MB Eindhoven, \\ Netherlands
}

\begin{abstract}
In this study, we investigated if hydrophobic particles of the materials carbon black and zinc oxide act as cavitation nuclei, using high-speed photography during 1-MHz, low-amplitude sonication. Carbon black particles were observed to lose their gaseous shells and become acoustically inactive, whilst zinc oxide particles were observed to generate bubbles without becoming acoustically inactive. Zinc oxide might be a suitable candidate for an ultrasound contrast agent. Carbon black might be a suitable candidate for ultrasound-guided drug delivery.
\end{abstract}

Ultrasonic technology is commonly used for medical imaging, ${ }^{1)}$ but it is also used to manipulate, ${ }^{2)}$ deform, ${ }^{3)}$ and disrupt particles with microscopic sizes. ${ }^{4)}$ Straightforward applications of particle manipulation are in separation processes such as wastewater treatment ${ }^{2)}$ and battery recycling. ${ }^{5)}$ The opposite of separation, i.e., mixing, has also been done with the aide of ultrasound. It was recently found, that ultrasound can even rapidly disperse nanoparticles, a process which has been associated with inertial cavitation and acoustic streaming. ${ }^{6}$ )

Hand-held ultrasonic devices mix small quantities whilst requiring the ultrasonic tip to be dipped in the liquid to be mixed, which may be undesirable. ${ }^{7)}$ Ultrasound cleaning baths, commonly used for cleaning metal objects, ${ }^{8)}$ have been proposed as convenient replacement of acoustic shakers for bulk mixing. ${ }^{9)}$

Hydrophobic particles inherently resist being suspended in water. Hydrophobic particles might be regarded as tiny solid particles surrounded by a thin gaseous shell. ${ }^{10)}$ It has been hypothesised that hydrophobic particles act as cavitation nuclei. ${ }^{10)}$ This cavitation behaviour would explain the translation speeds observed when hydrophobic polystyrene microspheres

*E-mail: michiel.postema@wits.ac.za 
were driven through a liquid medium by means of ultrasound. ${ }^{11)}$ These translation speeds corresponded to those observed with gas microbubbles of similar sizes. ${ }^{11)}$ If hydrophobic particles do have a thin gaseous layer surrounding the solid cores, a sound field of sufficient pressure amplitude must force the gas layer to form and inertial cavity which must subsequently fragment during the collapse phase. Such behaviour has been observed form other cavitation nuclei, using high-speed photography. ${ }^{4,12,13)}$

The effect of an ultrasonic cleaner on suspensions with hydrophobic particles was recently tested on two materials. ${ }^{14)}$ The first, carbon black, is a common particle in lithium ion battery cathodes, but also in tattoo ink. Hydrophobic carbon black has been observed to generate a harmonic response to a low-amplitude, i.e., non-destructive, ultrasound field. ${ }^{5)}$ The second material, hydrophobically modified zinc oxide, builds the endeskeleon of acoustically active antibubbles. ${ }^{15)}$ Vials with both materials before and after 1-minute sonication in an ultrasonic bath operating at $45 \mathrm{kHz}$ have been shown in Ref. 14. The particles of both materials were suspended optically homogeneously.

We hypothesised that hydrophobic particles act as cavitation nuclei that lose (part of) their gaseous layer during sonication. In this study, we investigated this hypothesis using high-speed photography. As opposed to Ref. 14, here we present full recordings for events using both carbon black and zinc oxide. In addition, multiple bursts were subjected to the same sample, to investigate if the remainder materials still act as cavitation nuclei.

Two-materials were studied using identical protocols. The first material studied was TIMCAL SUPER C65 Carbon Black (EQ-Lib-SuperC65) conductive additive (MTI Corportation, Richmond, CA, USA), hereafter referred to as C65. The second material studied was hydrophobically modified Zano 10 Plus zinc oxide (Umicore, Brussel, Belgium), hereafter referred to as $\mathrm{ZnO}$. For each material, samples of $1 \mathrm{mg}$ were deposited into a FALCON ${ }^{\circledR}$ 15 mL High-Clarity Polypropylene Conical Tube (Corning Science México S.A. de C.V., Reynosa, Tamaulipas, Mexico), after which $5 \mathrm{~mL}$ of 049-16787 Distilled Water (FUJIFILM Wako Pure Chemical Corporation, Chuo-Ku, Osaka, Japan) was added.

Tubes were either or shaken by hand for 5 minutes, or individually held by hand for 1 minute in a 2510J-MT BRANSONIC ${ }^{\circledR}$ ULTRASONIC CLEANER (BRANSON ULTRASONICS CORPORATION, Danbury, CT, USA) filled with degassed water and operating at a transmitting frequency of $45 \mathrm{kHz}$. From each tube, $200 \mu \mathrm{L}$ was pipetted into the observation chamber of a high-speed observation system. ${ }^{16)}$ The observation chamber was placed under an Eclipse Ti inverted microscope (Nikon Corporation, Minato-ku, Tokyo, Japan) with a Plan Apo LWD 40× WI (N.A. 1.15) objective lens. Attached to the microscope was an HPV-X2 
high-speed camera (Shimadzu, Nakagyo-ku, Kyoto, Japan), operating at 2 or 10 million frames per second. ${ }^{17)}$ Exposure times corresponded to $500 \mathrm{~ns}$ and $100 \mathrm{~ns}$ per frame, respectively. During camera recording, the materials were subjected to ultrasound pulses.

The pulses consisted of either 3 or 100 cycles, each at a centre transmitting frequency of $1 \mathrm{MHz}$ and a peak-negative pressure of $200 \mathrm{kPa}$, from a laboratory-assembled single-element transducer. ${ }^{16,17)}$ The time between subsequent pulses was at least 1 minute. The signal fed into the transducer was generated by an AFG320 arbitrary function generator (Sony-Tektronix, Shinagawa-ku, Tokyo, Japan) and amplified by a UOD-WB-1000 wide-band power amplifier (TOKIN Corporation, Shiroishi, Miyagi, Japan).

Figure 1 shows the first 64 frames of a representative example from 56 high-speed video recordings of $\mathrm{C} 65$ and $\mathrm{ZnO}$, each consisting of 256 frames. The high-speed video in Figure 1 was recorded at 10 million frames per second, during a 3-cycle burst. The frames shows a manually suspended hydrophobic C65 particle before, during, and after 1-MHz sonication. During sonication, an inertial cavity can be clearly seen. After sonication, the cavity is seen to be detached and located to the upper right of the deformed or fragmented particle. In later frames (not shown), the cavity is seen to have dissolved, leaving the deformed or fragmented C65 particle. During subsequent ultrasound pulses, the C65 particle remained unaffected (not shown). For all experiments with manually suspended C65, during the first pulse, the hydrophobic particles were observed to grow into inertial cavities, forcing the gas layer surrounding the particle to be released and dissolved. After the first pulse, no further cavitation activity was observed. This indicates that sonication can remove the thin gas layer surrounding a hydrophobic particle. This is shown in Figure 2, which consists of frames from three subsequent 100-cycle pulses. The high-speed video in Figure 2 was recorded at 2 million frames per second. The frames were selected from the full video, during expansion maxima. Only during the first burst, C65 particles are seen to act as cavitation nuclei. The second burst shows some cavitation activity below the focal plane (second-last frame). No cavitation activity can be observed during the third burst. Only the manually suspended C65 particles show cavitation activity, whereas not a single recording of C65 suspended with the ultrasonic cleaner shows cavitation activity or particle translation. It appears that a single burst of low-amplitude ultrasound is enough to change the hydrophobic nature of the C65 particles.

Figure 3 shows ultrasonically suspended $\mathrm{ZnO}$ particles in frames from three subsequent 100-cycle pulses. The high-speed video in Figure 3 was recorded with the same settings as Figure 2. Here, as opposed to C65, the particles keep on generating inertial cavitation bubbles during subsequent pulses. These are seen to have moved in the direction of the sound field. 


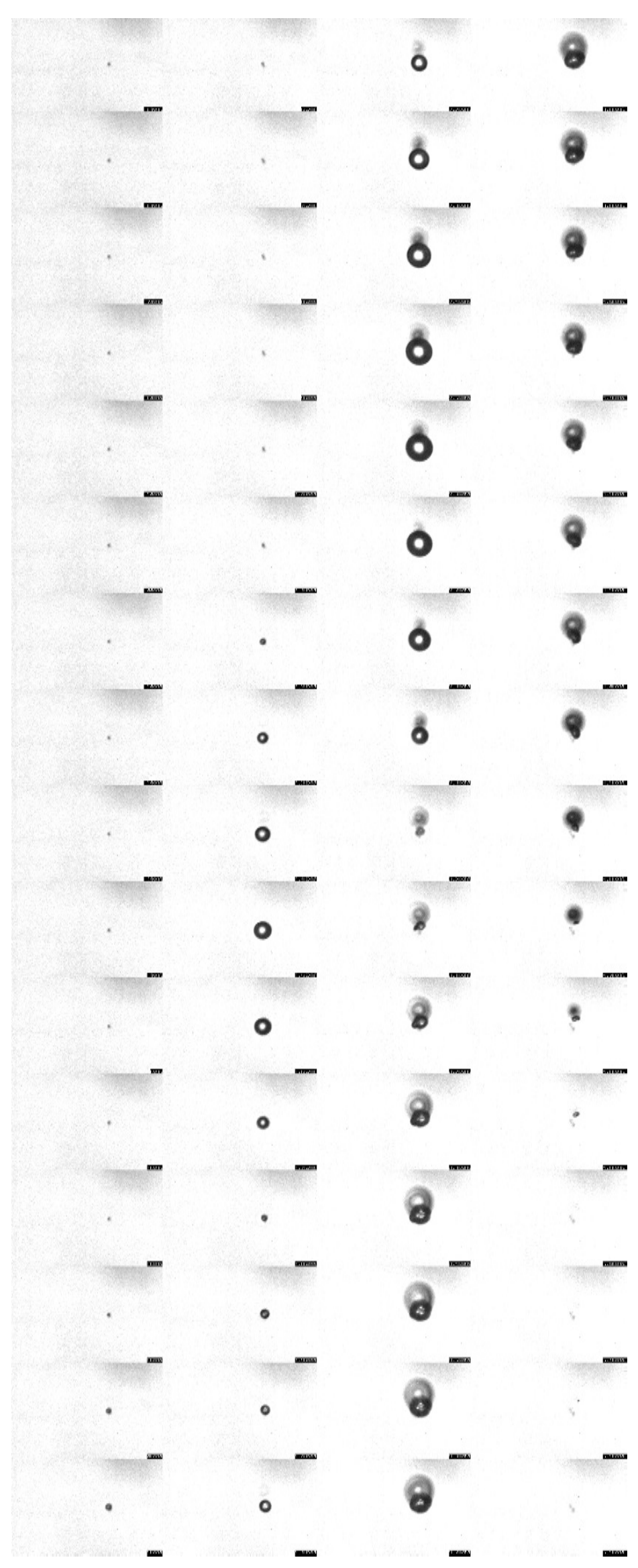

Fig. 1. C65 particle during a 3-cycle burst. Frames are ordered top-down, left-right. Time between frames is $100 \mathrm{~ns}$. Frame width is $145 \mu \mathrm{m}$. 


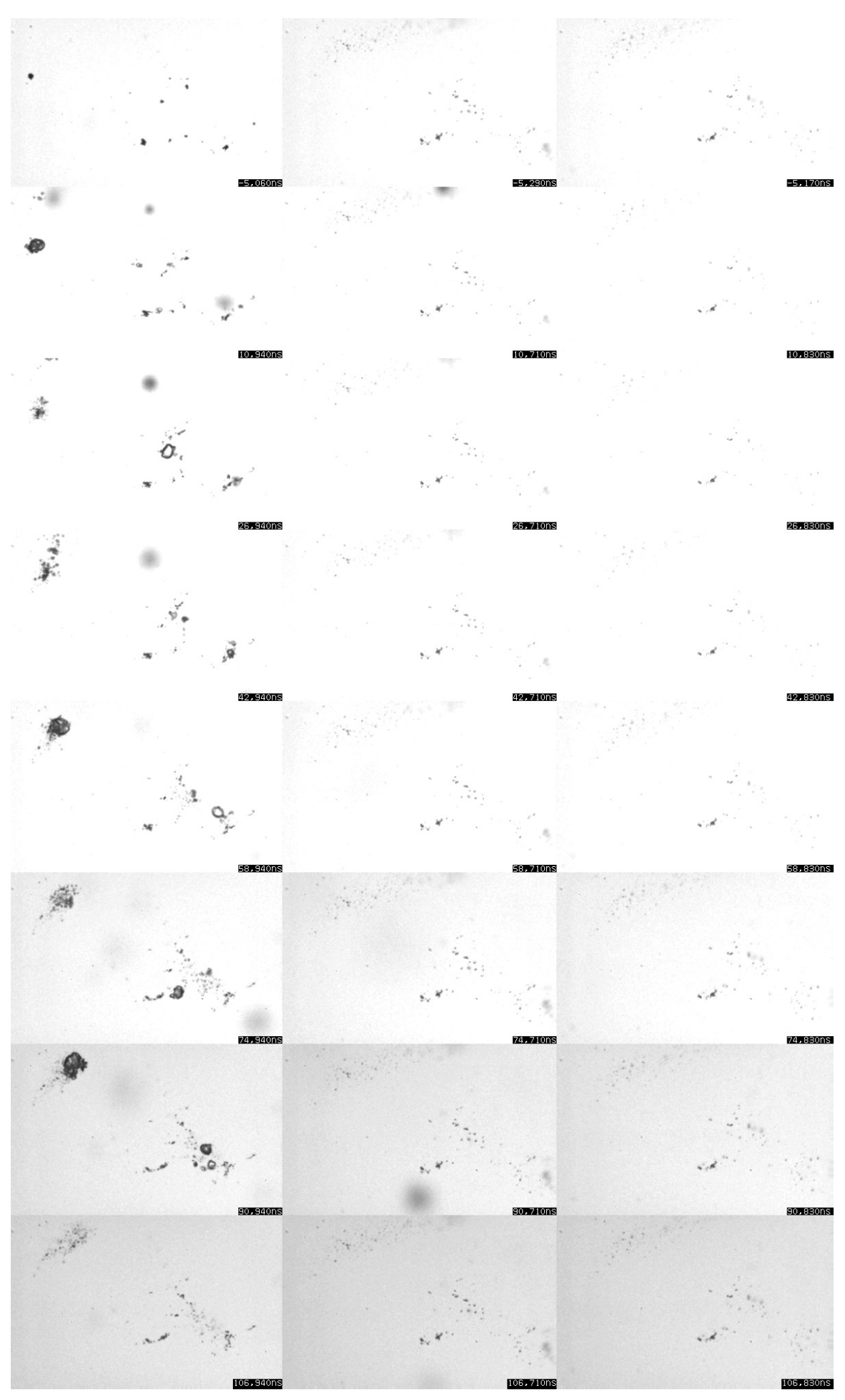

Fig. 2. C65 during three separate 100-cycle bursts (left-middle-right column). Frames are ordered top-down, left-right. Time between frames is $16 \mu$ s. Frame width is $145 \mu \mathrm{m}$. 


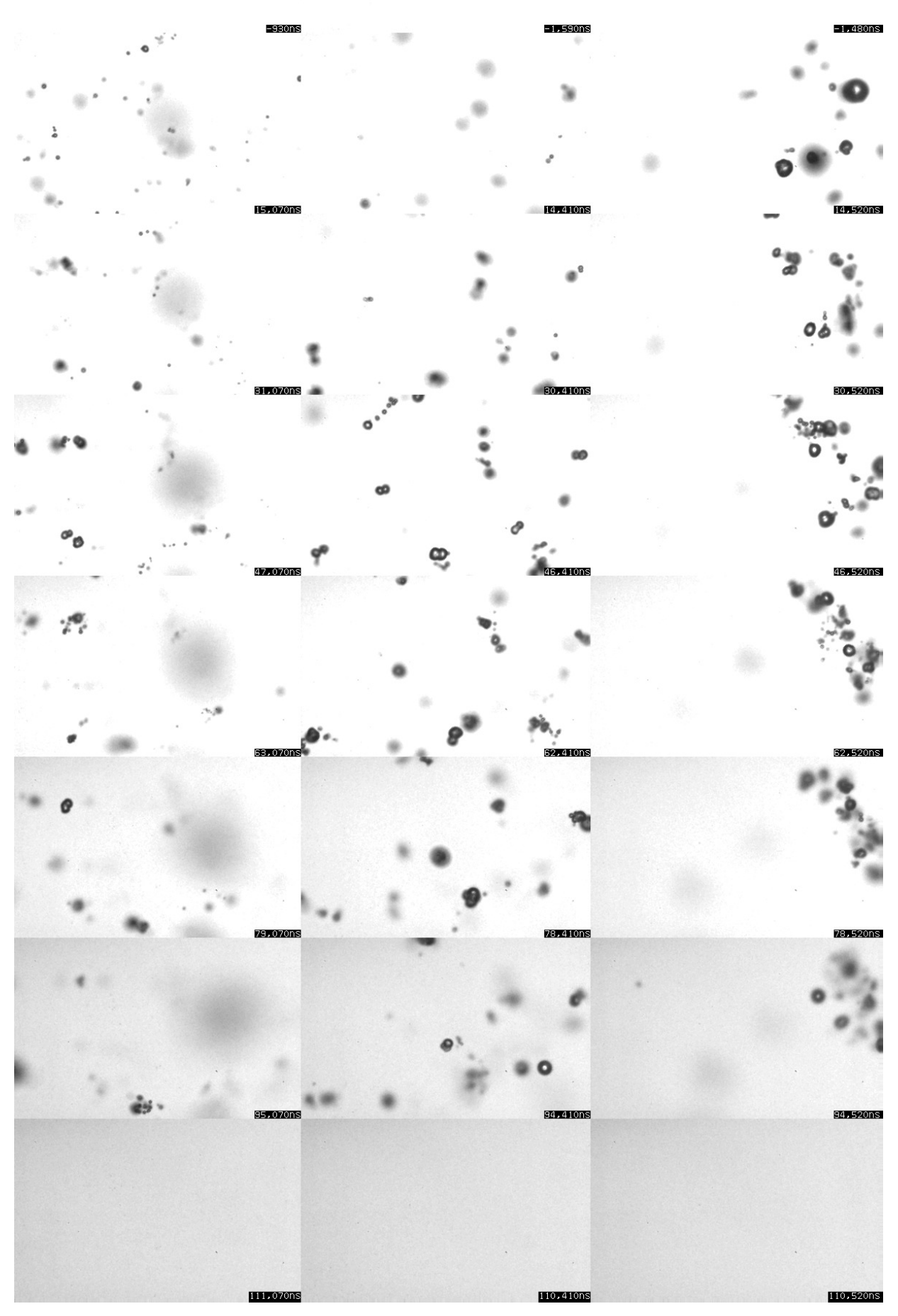

Fig. 3. $\mathrm{ZnO}$ during three separate 100-cycle bursts (left-middle-right column). Frames are ordered top-down, left-right. Time between frames is $16 \mu$ s. Frame width is $145 \mu \mathrm{m}$. 
$\mathrm{ZnO}$ does not lose its acoustic activity after sonication at low amplitudes.

Forced suspension of hydrophobic particles would be of major interest in ultrasoundguided drug delivery. The ultimate goal might be ultrasound-guided anticancer drugs. ${ }^{18)}$ If therapeutic compounds could be made hydrophobic, they would not interact with their (fluid) surroundings after injection. Only after turning them hydrophilic, with the aide of ultrasound, would they dissolve and act. Another option might be to let hydrophobic particles interact with cells in a similar way as microbubbles can be forced to interact with cells. ${ }^{19,20)}$

Hydrophobic particles of the materials carbon black and zinc oxide can be forced to be suspended in water using ultrasound. The high-speed observations shown in this paper confirm that hydrophobic particles can act as cavitation nuclei. The lack of cavitation from carbon black after the first pulse indicates that the gas layer surrounding the hydrophobic particle dissolves after inertial cavitation. Zinc oxide remains acoustically active after sonication. It might be a suitable candidate for an ultrasound contrast agent.

\section{Acknowledgement}

This work was supported by JSPS KAKENHI Grant Number 17H00864. 


\section{References}

1) S. Ishikura, M. Yoshizawa, N. Tagawa, and T. Irie, Jpn. J. Appl. Phys. 57 07LF20 (2018).

2) T. Kambayashi, T. Saeki, and I. Buchanan, Jpn. J. Appl. Phys. $5607 J E 04$ (2017).

3) J. Kim, J. Kim, J, Yeom, K. Ha, and M. Kim, Jpn. J. Appl. Phys. $5607 J D 04$ (2017).

4) S. Nishitaka, D. Mashiko, S. Yoshizawa, and S. Umemura, Jpn. J. Appl. Phys. 57 07LF25 (2018).

5) M. Postema, S. Phadke, A. Novell, R. Uzbekov, C. Nyamupangedengu, M. Anouti, and A. Bouakaz, Proc. 2019 IEEE AFRICON (2019).

6) M. Kim and J. Kim, Jpn. J. Appl. Phys. 57 07LE03 (2018).

7) Y. Qiu, H. Wang, C. E. Demore, D. A. Hughes, P. Glynne-Jones, S. Gebhardt, A. Bolhovitins, R. Poltarjonoks, K. Weijer, A. Schönecker, M. Hill, and S. Cochran, Sensors 1414806 (2014).

8) P. Gehrke, R. Smeets, M. Gosau, R. E. Friedrich, E. Madani, D. Duddeck, C. Fischer, F. Tebbel, R. Sader, and P. Hartjen, In Vivo 33689 (2019).

9) C. Shi, W. Yang, J. Chen, X. Sun, W. Chen, H. An, Y. Duo, and M. Pei, Ultrason. Sonochem. 37648 (2017).

10) P. Attard, Adv. Colloid Interface Sci. 10475 (2003).

11) N. Mazzawi, M. Postema, and E. Kimmel, Acta Phys. Polon. A. 127103 (2015).

12) K. Suzuki, R. Iwasaki, R. Takagi, S. Yoshizawa, and S. Umemura, Jpn. J. Appl. Phys. 56 07JF27 (2017).

13) N. Okada, M. Shiiba, S. Yamauchi, T. Sato, and S. Takeuchi, Jpn. J. Appl. Phys. 57 07LE15 (2018).

14) M. Postema, R. Matsumoto, R. Shimizu, A. T. Poortinga, and N. Kudo, Proc. 40th Symp. UltraSon. Electron. 2E2-4 (2019).

15) N. Kudo, R. Uzbekov, R. Matsumoto, R. Shimizu, C. Carlson, N. Anderton, A. Deroubaix, C. Penny, A. T. Poortinga, D. M. Rubin, A. Bouakaz, and M. Postema, Proc. 40th Symp. UltraSon. Electron. 2E3-1 (2019).

16) N. Kudo, IEEE Trans. Ultrason. Ferroelect. Freq. Control 64273 (2017).

17) S. Imai and N. Kudo, IEEE Int. Ultrason. Symp. IUS2018 184 (2018).

18) R. H. Rahayu, K. Takanashi, T. T. K. Soon, I. Seviaryna, R. Maev, K. Kobayashi, N. Hozumi, and S. Yoshida, Jpn. J. Appl. Phys. 56 07LF26 (2017).

19) R. Oitate, A. Shimomura, H. Wada, T. Mochizuki, K. Masuda, Y. Oda, R. Suzuki, and K. Maruyama, Jpn. J. Appl. Phys. 56 07JF25 (2017). 
20) R. Oitate, T. Otsuka, M. Seki, A. Furutani, T. Mochizuki, K. Masuda, R. Suzuki, and K. Murayama, Jpn. J. Appl. Phys. 57 07LF10 (2018). 


\title{
High-speed footage shows transient ultrasonic nucleation of different hydrophobic particles in suspension
}

\author{
Michiel Postema ${ }^{1 *}$, Ryonosuke Matsumoto ${ }^{2}$, Ri-ichiro Shimizu ${ }^{2}$, Albert T. Poortinga $^{3}$, and \\ Nobuki Kudo ${ }^{2}$ \\ ${ }^{1}$ School of Electrical and Information Engineering, University of the Witwatersrand, Johannesburg, \\ 1 Jan Smuts Laan, 2001 Braamfontein, South Africa \\ ${ }^{2}$ Faculty of Information Science and Technology, Hokkaido University, Kita 9 Chome, Nishi 14 Jo, Kita-ku, \\ Sapporo, Hokkaido 060-0814, Japan \\ ${ }^{3}$ Department of Mechanical Engineering, Eindhoven University of Technology, De Zaale, 5600 MB Eindhoven, \\ Netherlands
}

\begin{abstract}
In this study, we investigated if hydrophobic particles of the materials carbon black and zinc oxide act as cavitation nuclei, using high-speed photography during 1-MHz, low-amplitude sonication. Carbon black particles were observed to lose their gaseous shells and become acoustically inactive, whilst zinc oxide particles were observed to generate bubbles without becoming acoustically inactive. Zinc oxide might be a suitable candidate for an ultrasound contrast agent. Carbon black might be a suitable candidate for ultrasound-guided drug delivery.
\end{abstract}

Ultrasonic technology is commonly used for medical imaging, ${ }^{1)}$ but it is also used to manipulate, ${ }^{2)}$ deform, ${ }^{3)}$ and disrupt particles with microscopic sizes. ${ }^{4)}$ Straightforward applications of particle manipulation are in separation processes such as wastewater treatment ${ }^{2)}$ and battery recycling. ${ }^{5)}$ The opposite of separation, i.e., mixing, has also been done with the aide of ultrasound. It was recently found, that ultrasound can even rapidly disperse nanoparticles, a process which has been associated with inertial cavitation and acoustic streaming. ${ }^{6}$ )

Hand-held ultrasonic devices mix small quantities whilst requiring the ultrasonic tip to be dipped in the liquid to be mixed, which may be undesirable. ${ }^{7)}$ Ultrasound cleaning baths, commonly used for cleaning metal objects, ${ }^{8)}$ have been proposed as convenient replacement of acoustic shakers for bulk mixing. ${ }^{9)}$

Hydrophobic particles inherently resist being suspended in water. Hydrophobic particles might be regarded as tiny solid particles surrounded by a thin gaseous shell. ${ }^{10)}$ It has been hypothesised that hydrophobic particles act as cavitation nuclei. ${ }^{10)}$ This cavitation behaviour would explain the translation speeds observed when hydrophobic polystyrene microspheres

*E-mail: michiel.postema@wits.ac.za 
were driven through a liquid medium by means of ultrasound. ${ }^{11)}$ These translation speeds corresponded to those observed with gas microbubbles of similar sizes. ${ }^{11)}$ If hydrophobic particles do have a thin gaseous layer surrounding the solid cores, a sound field of sufficient pressure amplitude must force the gas layer to form and inertial cavity which must subsequently fragment during the collapse phase. Such behaviour has been observed form other cavitation nuclei, using high-speed photography. ${ }^{4,12,13)}$

The effect of an ultrasonic cleaner on suspensions with hydrophobic particles was recently tested on two materials. ${ }^{14)}$ The first, carbon black, is a common particle in lithium ion battery cathodes, but also in tattoo ink. Hydrophobic carbon black has been observed to generate a harmonic response to a low-amplitude, i.e., non-destructive, ultrasound field. ${ }^{5)}$ The second material, hydrophobically modified zinc oxide, builds the endeskeleon of acoustically active antibubbles. ${ }^{15)}$ Vials with both materials before and after 1-minute sonication in an ultrasonic bath operating at $45 \mathrm{kHz}$ have been shown in Ref. 14. The particles of both materials were suspended optically homogeneously.

We hypothesised that hydrophobic particles act as cavitation nuclei that lose (part of) their gaseous layer during sonication. In this study, we investigated this hypothesis using high-speed photography. As opposed to Ref. 14, here we present full recordings for events using both carbon black and zinc oxide. In addition, multiple bursts were subjected to the same sample, to investigate if the remainder materials still act as cavitation nuclei.

Two-materials were studied using identical protocols. The first material studied was TIMCAL SUPER C65 Carbon Black (EQ-Lib-SuperC65) conductive additive (MTI Corportation, Richmond, CA, USA), hereafter referred to as C65. The second material studied was hydrophobically modified Zano 10 Plus zinc oxide (Umicore, Brussel, Belgium), hereafter referred to as $\mathrm{ZnO}$. For each material, samples of $1 \mathrm{mg}$ were deposited into a FALCON ${ }^{\circledR}$ 15 mL High-Clarity Polypropylene Conical Tube (Corning Science México S.A. de C.V., Reynosa, Tamaulipas, Mexico), after which $5 \mathrm{~mL}$ of 049-16787 Distilled Water (FUJIFILM Wako Pure Chemical Corporation, Chuo-Ku, Osaka, Japan) was added.

Tubes were either or shaken by hand for 5 minutes, or individually held by hand for 1 minute in a 2510J-MT BRANSONIC ${ }^{\circledR}$ ULTRASONIC CLEANER (BRANSON ULTRASONICS CORPORATION, Danbury, CT, USA) filled with degassed water and operating at a transmitting frequency of $45 \mathrm{kHz}$. From each tube, $200 \mu \mathrm{L}$ was pipetted into the observation chamber of a high-speed observation system. ${ }^{16)}$ The observation chamber was placed under an Eclipse Ti inverted microscope (Nikon Corporation, Minato-ku, Tokyo, Japan) with a Plan Apo LWD 40× WI (N.A. 1.15) objective lens. Attached to the microscope was an HPV-X2 
high-speed camera (Shimadzu, Nakagyo-ku, Kyoto, Japan), operating at 2 or 10 million frames per second. ${ }^{17)}$ Exposure times corresponded to 500 ns and 100 ns per frame, respectively. During camera recording, the materials were subjected to ultrasound pulses.

The pulses consisted of either 3 or 100 cycles, each at a centre transmitting frequency of $1 \mathrm{MHz}$ and a peak-negative pressure of $200 \mathrm{kPa}$, from a laboratory-assembled single-element transducer. ${ }^{16,17)}$ The time between subsequent pulses was at least 1 minute. The signal fed into the transducer was generated by an AFG320 arbitrary function generator (Sony-Tektronix, Shinagawa-ku, Tokyo, Japan) and amplified by a UOD-WB-1000 wide-band power amplifier (TOKIN Corporation, Shiroishi, Miyagi, Japan).

Figure 1 shows the first 64 frames of a representative example from 56 high-speed video recordings of $\mathrm{C} 65$ and $\mathrm{ZnO}$, each consisting of 256 frames. The high-speed video in Figure 1 was recorded at 10 million frames per second, during a 3-cycle burst. The frames shows a manually suspended hydrophobic C65 particle before, during, and after 1-MHz sonication. During sonication, an inertial cavity can be clearly seen. After sonication, the cavity is seen to be detached and located to the upper right of the deformed or fragmented particle. In later frames (not shown), the cavity is seen to have dissolved, leaving the deformed or fragmented C65 particle. During subsequent ultrasound pulses, the C65 particle remained unaffected (not shown). For all experiments with manually suspended C65, during the first pulse, the hydrophobic particles were observed to grow into inertial cavities, forcing the gas layer surrounding the particle to be released and dissolved. After the first pulse, no further cavitation activity was observed. This indicates that sonication can remove the thin gas layer surrounding a hydrophobic particle. This is shown in Figure 2, which consists of frames from three subsequent 100-cycle pulses. The high-speed video in Figure 2 was recorded at 2 million frames per second. The frames were selected from the full video, during expansion maxima. Only during the first burst, C65 particles are seen to act as cavitation nuclei. The second burst shows some cavitation activity below the focal plane (second-last frame). No cavitation activity can be observed during the third burst. Only the manually suspended C65 particles show cavitation activity, whereas not a single recording of C65 suspended with the ultrasonic cleaner shows cavitation activity or particle translation. It appears that a single burst of low-amplitude ultrasound is enough to change the hydrophobic nature of the C65 particles.

Figure 3 shows ultrasonically suspended $\mathrm{ZnO}$ particles in frames from three subsequent 100-cycle pulses. The high-speed video in Figure 3 was recorded with the same settings as Figure 2. Here, as opposed to C65, the particles keep on generating inertial cavitation bubbles during subsequent pulses. These are seen to have moved in the direction of the sound field. 


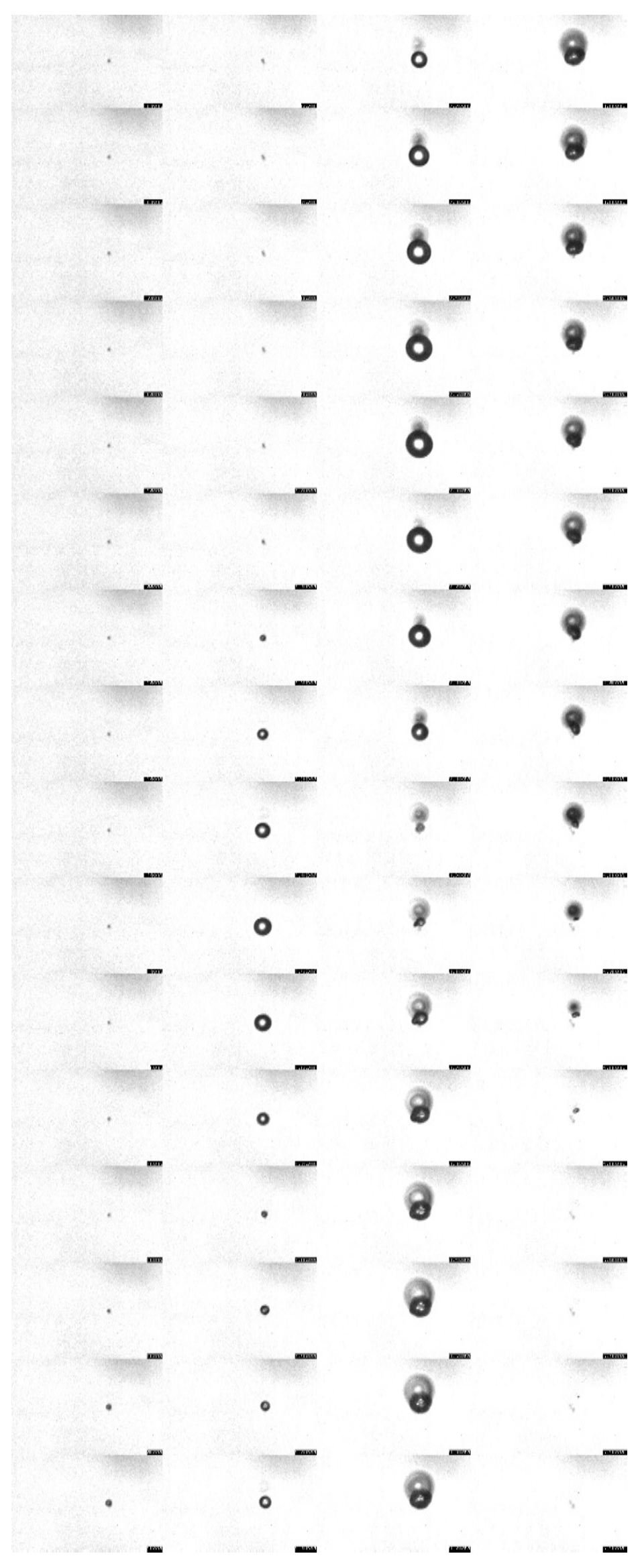

Fig. 1. C65 particle during a 3-cycle burst. Frames are ordered top-down, left-right. Time between frames is $100 \mathrm{~ns}$. Frame width is $145 \mu \mathrm{m}$. 


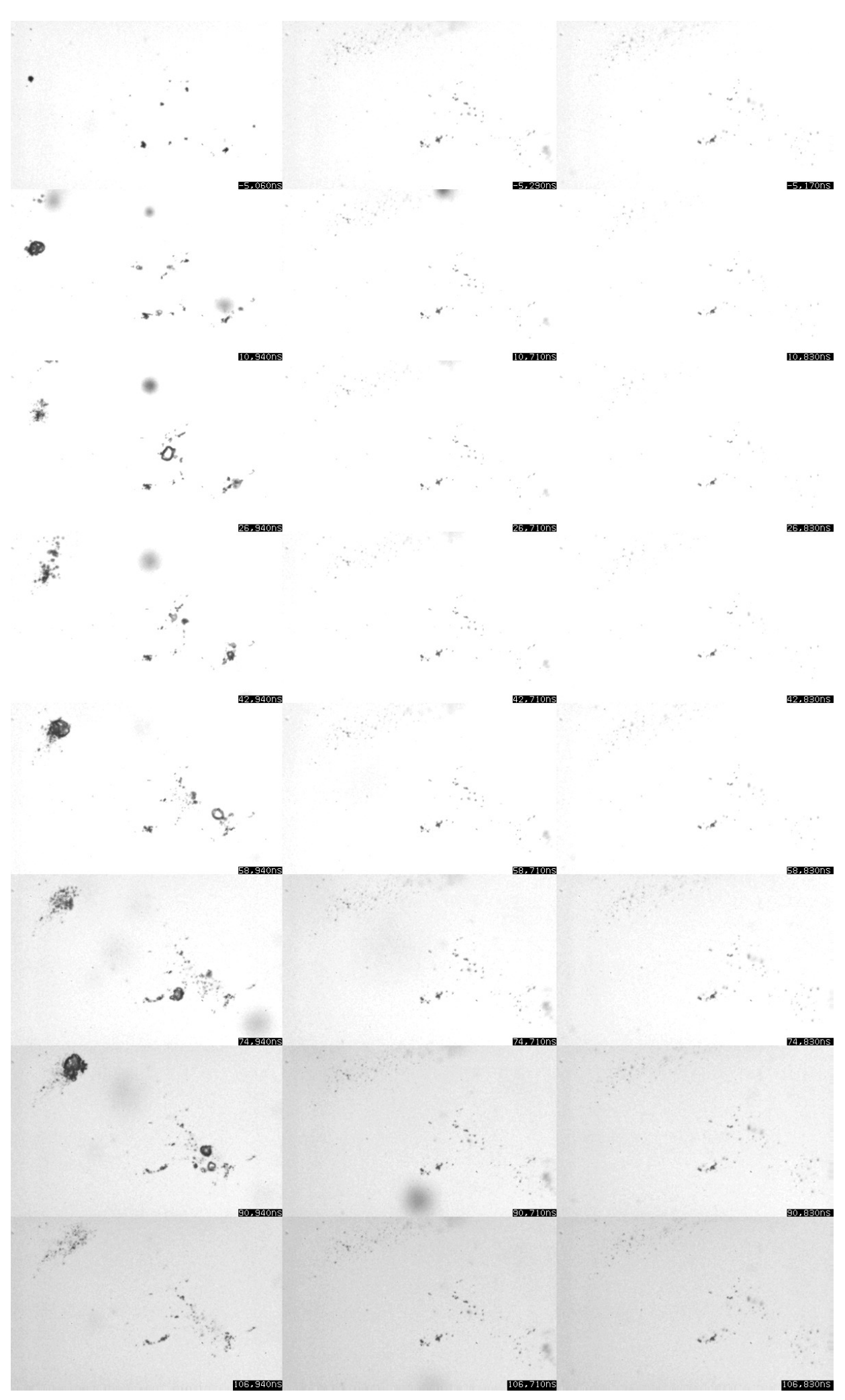

Fig. 2. C65 during three separate 100-cycle bursts (left-middle-right column). Frames are ordered top-down, left-right. Time between frames is $16 \mu \mathrm{s}$. Frame width is $145 \mu \mathrm{m}$. 


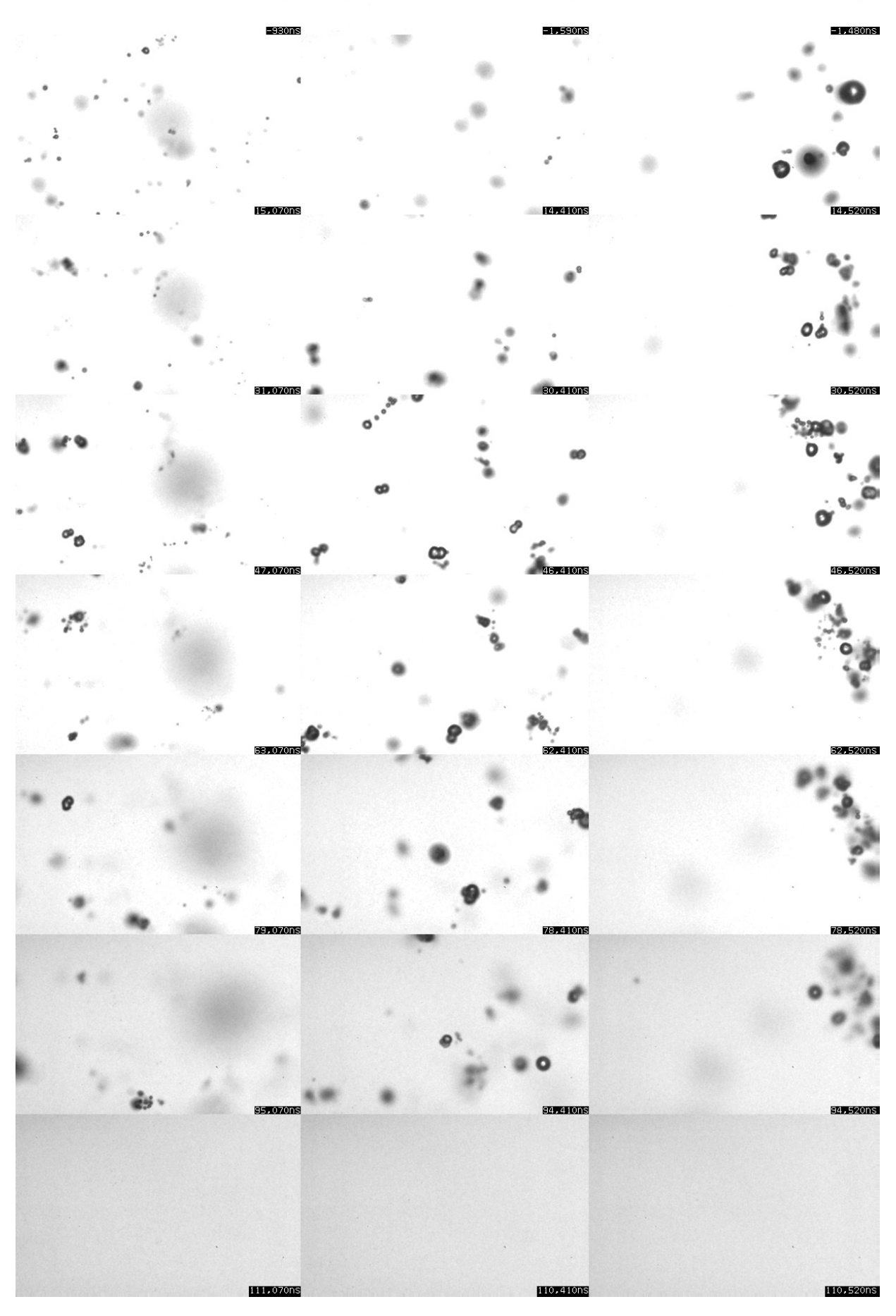

Fig. 3. ZnO during three separate 100-cycle bursts (left-middle-right column). Frames are ordered top-down, left-right. Time between frames is $16 \mu \mathrm{s}$. Frame width is $145 \mu \mathrm{m}$. 
$\mathrm{ZnO}$ does not lose its acoustic activity after sonication at low amplitudes.

Forced suspension of hydrophobic particles would be of major interest in ultrasoundguided drug delivery. The ultimate goal might be ultrasound-guided anticancer drugs. ${ }^{18)}$ If therapeutic compounds could be made hydrophobic, they would not interact with their (fluid) surroundings after injection. Only after turning them hydrophilic, with the aide of ultrasound, would they dissolve and act. Another option might be to let hydrophobic particles interact with cells in a similar way as microbubbles can be forced to interact with cells. ${ }^{19,20)}$

Hydrophobic particles of the materials carbon black and zinc oxide can be forced to be suspended in water using ultrasound. The high-speed observations shown in this paper confirm that hydrophobic particles can act as cavitation nuclei. The lack of cavitation from carbon black after the first pulse indicates that the gas layer surrounding the hydrophobic particle dissolves after inertial cavitation. Zinc oxide remains acoustically active after sonication. It might be a suitable candidate for an ultrasound contrast agent.

\section{Acknowledgement}

This work was supported by JSPS KAKENHI Grant Number 17H00864. 


\section{References}

1) S. Ishikura, M. Yoshizawa, N. Tagawa, and T. Irie, Jpn. J. Appl. Phys. 57 07LF20 (2018).

2) T. Kambayashi, T. Saeki, and I. Buchanan, Jpn. J. Appl. Phys. $5607 J E 04$ (2017).

3) J. Kim, J. Kim, J, Yeom, K. Ha, and M. Kim, Jpn. J. Appl. Phys. $5607 J D 04$ (2017).

4) S. Nishitaka, D. Mashiko, S. Yoshizawa, and S. Umemura, Jpn. J. Appl. Phys. 57 07LF25 (2018).

5) M. Postema, S. Phadke, A. Novell, R. Uzbekov, C. Nyamupangedengu, M. Anouti, and A. Bouakaz, Proc. 2019 IEEE AFRICON (2019).

6) M. Kim and J. Kim, Jpn. J. Appl. Phys. 57 07LE03 (2018).

7) Y. Qiu, H. Wang, C. E. Demore, D. A. Hughes, P. Glynne-Jones, S. Gebhardt, A. Bolhovitins, R. Poltarjonoks, K. Weijer, A. Schönecker, M. Hill, and S. Cochran, Sensors 1414806 (2014).

8) P. Gehrke, R. Smeets, M. Gosau, R. E. Friedrich, E. Madani, D. Duddeck, C. Fischer, F. Tebbel, R. Sader, and P. Hartjen, In Vivo 33689 (2019).

9) C. Shi, W. Yang, J. Chen, X. Sun, W. Chen, H. An, Y. Duo, and M. Pei, Ultrason. Sonochem. 37648 (2017).

10) P. Attard, Adv. Colloid Interface Sci. 10475 (2003).

11) N. Mazzawi, M. Postema, and E. Kimmel, Acta Phys. Polon. A. 127103 (2015).

12) K. Suzuki, R. Iwasaki, R. Takagi, S. Yoshizawa, and S. Umemura, Jpn. J. Appl. Phys. 56 07JF27 (2017).

13) N. Okada, M. Shiiba, S. Yamauchi, T. Sato, and S. Takeuchi, Jpn. J. Appl. Phys. 57 07LE15 (2018).

14) M. Postema, R. Matsumoto, R. Shimizu, A. T. Poortinga, and N. Kudo, Proc. 40th Symp. UltraSon. Electron. 2E2-4 (2019).

15) N. Kudo, R. Uzbekov, R. Matsumoto, R. Shimizu, C. Carlson, N. Anderton, A. Deroubaix, C. Penny, A. T. Poortinga, D. M. Rubin, A. Bouakaz, and M. Postema, Proc. 40th Symp. UltraSon. Electron. 2E3-1 (2019).

16) N. Kudo, IEEE Trans. Ultrason. Ferroelect. Freq. Control 64273 (2017).

17) S. Imai and N. Kudo, IEEE Int. Ultrason. Symp. IUS2018 184 (2018).

18) R. H. Rahayu, K. Takanashi, T. T. K. Soon, I. Seviaryna, R. Maev, K. Kobayashi, N. Hozumi, and S. Yoshida, Jpn. J. Appl. Phys. 56 07LF26 (2017).

19) R. Oitate, A. Shimomura, H. Wada, T. Mochizuki, K. Masuda, Y. Oda, R. Suzuki, and K. Maruyama, Jpn. J. Appl. Phys. 56 07JF25 (2017). 
20) R. Oitate, T. Otsuka, M. Seki, A. Furutani, T. Mochizuki, K. Masuda, R. Suzuki, and K. Murayama, Jpn. J. Appl. Phys. 57 07LF10 (2018). 